PROCEEDINGS OF THE

AMERICAN MATHEMATICAL SOCIETY

Volume 125, Number 7, July 1997, Pages 2161-2170

S 0002-9939(97)03768-4

\title{
AUSLANDER SYSTEMS
}

\author{
KAMEL N. HADDAD AND AIMEE S. A. JOHNSON
}

(Communicated by Mary Rees)

\begin{abstract}
The authors generalize the dynamical system constructed by J. Auslander in 1959, resulting in perhaps the simplest family of examples of minimal but not strictly ergodic systems. A characterization of unique ergodicity and mean-L-stability is given. The new systems are also shown to have zero topological entropy and fail to be weakly rigid. Some results on the set of idempotents in the enveloping semigroup are also achieved.
\end{abstract}

\section{INTRODUCTION}

In 1958, J. Auslander [1] introduced a minimal, mean-L-stable, yet non distal dynamical system which is a subset of the unit square and projects onto the triadic adding machine on the unit interval. This example was inspired by an example due to E. E. Floyd [4]. Auslander's example was provided with the intent to illustrate possible behaviours of mean-L-stable systems, and due to its intriguing properties, earned interest in its own right.

The construction leading to Auslander's example naturally lends itself to generalization. In this paper, we study a class of generalizations of this example which yield minimal topological systems we term Auslander systems. These systems are easy to describe and not difficult to visualize, yet they exhibit nontrivial dynamics which are explored in this paper. Several results are achieved including of note: On entropy: in [2], Auslander and Berg show that the entropy of Auslander's original example is zero. We show that this remains true for all Auslander systems. On unique ergodicity and mean-L-stability: We prove that Auslander systems can either be uniquely ergodic, or fail to be so, and we give a characterization of when unique ergodicity holds. As a consequence, some Auslander systems are meanL-stable and some are not. On the set of idempotents $J(X)$ in the enveloping semigroup: we compute $J(X)$ for a "well behaved" class of Auslander systems, resulting in a set of cardinality $c$ (the largest cardinality $J(X)$ could achieve in general is $2^{c}$ ), and give insight to the fact that in general Auslander systems, $J(X)$ could be quite complex (for a thorough and enlightning treatment on the role of the set of idempotents in the enveloping semigroup, see [3]). Despite the complexity of the enveloping semigroup for these systems, it is possible to prove that the identity function is never in the asymptotic enveloping semigroup of an Auslander system. So Auslander systems are never weakly rigid.

Received by the editors August 15, 1995 and, in revised form, January 16, 1996.

1991 Mathematics Subject Classification. Primary 54H20, 54H15.

Key words and phrases. Dynamical system, minimality, proximality, entropy, unique ergodicity, enveloping semigroup. 
The authors would like to thank Joseph Auslander and Kenneth Berg for the many fruitful discussions held during the preparation of this paper.

\section{The CONSTRUCTION}

Let $B_{0}=[0,1] \times[0,1]$. We say that $\alpha$ determines a subdivision pattern for $B_{0}$, given by a subset $\alpha\left(B_{0}\right)$ of $B_{0}$, if $\alpha\left(B_{0}\right)$ is a union of subrectangles of $B_{0}$, denoted by $B^{0}, B^{1}, \ldots, B^{n-1}$, such that for every superscript $i$,

- The width of $B^{i}$ equals $(2 n-1)^{-1}$,

- $B^{i}$ is centered about the axis $x=\frac{4 i+1}{2(2 n-1)}$,

- $B^{i}$ meets the axis $y=0$ or $y=1$,

- $B^{i}$ has height 1 or $\frac{1}{2}$.

Say that $B^{i}$ is of type $\mathrm{F}$ (for full height) if the height of $B^{i}$ is 1 and of type $\mathrm{H}$ (for half height) if the height of $B^{i}$ is $\frac{1}{2}$.

We can then define $\alpha$ on any rectangle $R \subset B_{0}$ by superimposing $\alpha\left(B_{0}\right)$ onto $R$. This yields a union of subrectangles of $R$ which again can be described as of type $\mathrm{F}$ or $\mathrm{H}$ by analogy to $\alpha\left(B_{0}\right)$. For example, if $\alpha\left(B_{0}\right)$ is given by:

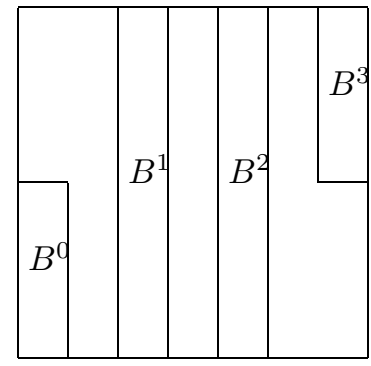

Unit Square

then $\alpha\left(B^{3}\right)$ is given by the shaded portion:

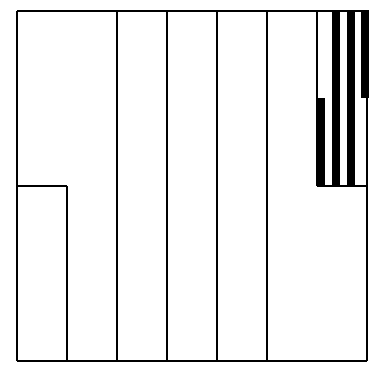

In the above figure, the two middle shaded rectangles are of type $\mathrm{F}$.

A fixed sequence $\left\{\alpha_{i}\right\}_{i \in \mathbf{N}}$ of subdivision patterns determines a dynamical system $(X, T)$ in the following inductive manner: let $n_{i}$ be the number of rectangles in $\alpha_{i}\left(B_{0}\right)$ and set $\nu_{j}=\prod_{k=1}^{j} n_{k}$. Let $B_{1}=\alpha_{1}\left(B_{0}\right)=B_{1}^{0} \cup B_{1}^{1} \cup \cdots \cup B_{1}^{n_{1}-1}$. Denote $\alpha_{j}\left(B_{j-1}^{k}\right)$ by $B_{j}^{k} \cup B_{j}^{k+\nu_{(j-1)}} \cup \cdots \cup B_{j}^{k+\left(n_{j}-1\right) \nu_{(j-1)}}$ and then set $B_{j}=$ $\cup_{k=0}^{\nu_{(j-1)}-1} \alpha_{j}\left(B_{j-1}^{k}\right)$, which can also be denoted, using the above, by $\cup_{k=0}^{\nu_{j}-1} B_{j}^{k}$. Notice that the superscripts of these rectangles $B_{j}^{k}$ no longer increase successively from 
left to right, although $B_{j}^{0}$ is always the leftmost rectangle and $B_{j}^{\nu_{j}-1}$ the rightmost. Finally, define $T_{j}$ on $B_{j}, j \in N$, as the linear map which sends $B_{j}^{k}$ to $B_{j}^{k+1}$ for $k<\nu_{j}-1$ and leaves $T_{j}$ undefined on $B_{j}^{\nu_{j}-1}$.

Let $X=\cap_{i \in N} B_{i}$. X is a compact space which is made up of lines and points, and inherits the Euclidean metric of the plane. From this point on, the term "line" will always be taken to mean a line with nonzero length, and the term "degenerate point" will be taken to mean a point in $X$ not lying on any line.

In order to define a continuous injective map $T$ on $X$ by $T=\lim _{i \rightarrow \infty} T_{i}$, we need to set some restrictions on our subdivision patterns. Continuity necessitates that $T$ map the line or degenerate point with $x$-coordinate 1 to the line or degenerate point with $x$-coordinate 0 . For $T$ to be injective and well defined, $X$ must then have a line over $x=1$ if and only if $X$ has a line over $x=0$. If these two are indeed lines, then to ensure continuity there must exist a $K$ such that for all $i \geq K, B_{i}^{m}$ is of type $\mathrm{F}$ for all $m$. Otherwise, $\alpha_{i}$ has both its first and last boxes of full length for $i \geq K$ but has other subboxes of half length, in which case one can find two sequences of points $(x, y)$ converging to $(1, y)$, one with $(x, y)$ lying on a subbox half the length of the other. The images under $T$ of these two sequences converge to different points. This violates the continuity of $T$. Note that the assumption that $B_{i}^{m}$ is of type $\mathrm{F}$ for all $i \geq K$ and all $m$ yields a system $(X, T)$ isomorphic to the direct product of the adding machine and $[0,1]$. Also, if there are infinitely many $i$ with $B_{i}^{m}$ of type $\mathrm{H}$ for all $m$, then $(X, T)$ will again be isomorphic to the adding machine. Since the dynamics of the adding machine are well known, we omit these two cases in our study.

Definition 2.1. If a sequence of subdivision patterns $\left\{\alpha_{i}\right\}_{i \in N}$ satisfies the properties:

- there are infinitely many $i$ with $B_{i}^{0}$ of type $\mathrm{H}$ and infinitely many $j$ with $B_{j}^{\nu_{j}-1}$ of type $\mathrm{H}$,

- at most finitely many $i$ have $B_{i}^{m}$ of type $\mathrm{H}$ for all $m$,

then the resulting system $(X, T)$ will be called an Auslander system.

Auslander systems are generalizations of the dynamical system introduced by J. Auslander in [1]. In the original Auslander example, all $\alpha_{i}$ are the same and determine the subdivision pattern shown below:

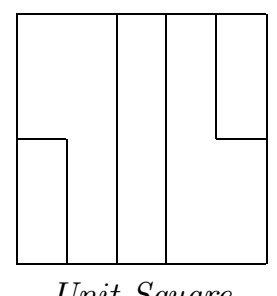

Unit Square

We may describe the points and lines of $X$ symbolically. Let $N(i)=\left\{0,1, \ldots, n_{i}-\right.$ 1 \} be a numbering of the subboxes of $\alpha_{i}\left(B_{0}\right)$ from left to right, and let $Q(i) \subset N(i)$ correspond to those subrectangles of type $\mathrm{F}$. If $(x, y) \in X$, we may describe $x$ uniquely by $x_{1} x_{2} x_{3} \ldots \ldots \in Y=\prod_{i=1}^{\infty} N(i)$ by setting $x_{j}=k \Leftrightarrow(x, y)$ belongs to the $(k+1)$ subrectangle of $\alpha_{j}\left(B_{j-1}^{m}\right)$. We will denote $x_{1} x_{2} x_{3} \ldots$ resulting from $x$ by $\bar{x}$. Also, if $L$ is a line in $X$ with $x$-coordinate $x$, we will have occasion to write $L \sim x_{1} x_{2} x_{3} \ldots$ The map $T$ on $X$ projects to the adding machine on $Y$, modulo $n_{i}$ at slot $i$, carrying to the right. 
The following two lemmas are consequences of the construction.

Lemma 2.2. Let $(X, T)$ be an Auslander system. Let $(x, y) \in X$ and let $\bar{x}=$ $x_{1} x_{2} x_{3} \ldots$ Then $(x, y)$ belongs to a line iff there exists $k$ such that for every $i \geq k$, $x_{i} \in Q(i)$.

Lemma 2.3. Let $(X, T)$ be an Auslander system. $X$ contains only countably many lines if and only if there exists $K$ such that for every $i \geq K,|Q(i)|=1$.

Now denote $Q(i)^{c}$ by $H(i)$ and divide $H(i)$ into $H_{L}(i)$ and $H_{U}(i)$, depending on whether the corresponding rectangle of $\alpha_{i}\left(B_{0}\right)$ lies in the lower or the upper half of $B_{0}$ respectively. Thus $N(i)=Q(i) \cup H_{L}(i) \cup H_{U}(i)$. The following two lemmas follow from writing $x$ in the symbolic form $\bar{x}$.

Lemma 2.4. If $\left\{i: x_{i} \in H(i)\right\}$ is finite, then $(x, y)$ belongs to a line $L$ whose length $l(L)$ is $\frac{1}{2^{k}}$ iff $\left|\left\{i: x_{i} \in H(i)\right\}\right|=k$. If $\left\{i: x_{i} \in H(i)\right\}$ is infinite, then $(x, y)$ is a degenerate point.

Lemma 2.5. If $(x, y)$ is a degenerate point, then $y$ can be found using $\bar{x}$ as stated below, and if $(x, y)$ lies on line $L$, then the ordinate $y_{b}$ of the base (bottom point) of $L$ can be found in the same way: delete from $\bar{x}$ all $x_{j}$ such that $x_{j} \in Q(j)$ and reindex the remaining sequence by $N$.

Let $M=\left\{i \in N: x_{i} \in H_{U}(i)\right\}$. Then $y$ (or $y_{b}$ ) equals $\sum_{n \in M} \frac{1}{2^{n}}$.

\section{Minimality}

Recall that for all points $(x, y) \in X$ except those with $\bar{x}=\left(n_{1}-1\right)\left(n_{2}-1\right) \ldots$, $T(x, y)$ is determined by some $T_{m}$.

Theorem 3.1. Let $(X, T)$ be an Auslander system. Then $T$ is continuous on $X$.

Proof. If there exists $i$ such that $x_{i} \neq n_{i}-1$ (so $(x, y)$ is not in the last rectangle at every stage), then at stage $i,(x, y)$ belongs to some $B_{i}^{k}$ which maps linearly under $T_{i}$ to rectangle $B_{i}^{k+1}$. Clearly $T$ is then continuous at $(x, y)$.

Otherwise $x=1$, and $(1, y)$ is a degenerate point. Let $J$ be such that $B_{J}^{0}$ and $B_{J}^{\nu_{J}-1}$ have height less than $\epsilon$. Let $\delta=\min \left\{\epsilon\right.$, width of $\left.B_{J}^{i}\right\}$. Then the $\delta \times \delta$ box surrounding $(x, y)$ is mapped within the $\epsilon \times \epsilon$ box surrounding $T(x, y)$.

Since $(X, T)$ projects to the adding machine, we can follow the orbit of the $x$ coordinate of a point by using the adding machine on $Y$. Proximality is thus easy to determine:

Theorem 3.2. Two points $\left(x_{1}, y_{1}\right)\left(x_{2}, y_{2}\right)$ in an Auslander system are proximal iff $x_{1}=x_{2}$ (and thus they belong to the same line).

The next lemma and theorem will show that all Auslander systems are minimal. We will denote the usual Euclidean distance on $X$ by $d($,$) , and if L$ is a line in $X$, we will use $y_{b}(L)$ to denote the $y$-coordinate of the bottom point of $L$.

Lemma 3.3. Let $(X, T)$ be an Auslander system satisfying the following condition: (C) Given a point on a line and a neighborhood $U$ of this point, we can find a degenerate point $(x, y) \in X$ such that $(x, y) \in U$.

Then $(X, T)$ is a minimal system. 
Proof. Let $(x, y)$ be an arbitrary point in $X$. We will show that the orbit closure of $(x, y)$ under $T$ is all of $X$.

The orbit of $(x, y)$ visits the neighborhood of any degenerate point since adding machines are minimal.

Otherwise, suppose that $\left(x^{\prime}, y^{\prime}\right)$ is a point on a line and let $U$ be a neighborhood of $\left(x^{\prime}, y^{\prime}\right)$. By condition $(\mathrm{C})$, there exists a degenerate point $\left(x_{1}, y_{1}\right)$ in $U$. But as before, the orbit of $(x, y)$ comes arbitrarily close to $\left(x_{1}, y_{1}\right)$.

Theorem 3.4. Let $(X, T)$ be an Auslander system. Then $(X, T)$ is minimal iff both of the following are satisfied:

i) There exists infinitely many $i$ with $\left|H_{U}(i)\right|>0$.

ii) There exists infinitely many $j$ with $\left|H_{L}(j)\right|>0$.

Proof. If the condition does not hold then either exactly one or neither of i) and ii) is satisfied. If neither is satisfied, then $(X, T)$ is not an Auslander system and besides, is clearly not minimal.

If i) is satisfied but not ii), then there will be a height $y_{0}>0$, below which no degenerate points exist. The set of degenerate points would then form a nonempty proper $T$-invariant subset of $X$. A similar situation arises if ii) is satisfied but not i).

Now assume i) and ii) are satisfied. To show $(X, T)$ is minimal we just need to show it satisfies condition (C) of Lemma 3.3.

Let $(x, y)$ belong to line $L$. Let $U$ be a $p \times p$ neighborhood of $(x, y)$ and $\bar{x}=$ $x_{1} x_{2} x_{3} \ldots$ Choose $\left(x^{\prime}, y^{\prime}\right)$ so that $\overline{x^{\prime}}=x_{1}^{\prime} x_{2}^{\prime} x_{3}^{\prime} \ldots$ and $x_{i}^{\prime}=x_{i}$ for $1 \leq i \leq I$, where $I$ is such that $\mathrm{d}\left(x, x^{\prime}\right)<p$. Choose $x_{i}^{\prime} \in H_{L}(i) \cup H_{U}(i)$ for $i>I$ in such a way that, by Lemma $2.5,\left(x^{\prime}, y^{\prime}\right)$ is a degenerate point in $U$.

\section{UNIQUE ERGODICITY AND TOPOLOGICAL ENTROPY}

Recall that the adding machine is uniquely ergodic and has entropy zero.

Thus any interesting dynamical phenomena must occur on the set of lines $\tilde{L}$ of $(X, T)$. Any ergodic measure $\mu$ on $(X, T)$ must project to the unique measure which gives each symbol equal weight. If $\mu(\tilde{L})=0$ then $X$ is measurably isomorphic to the adding machine and trivially $(X, T)$ is uniquely ergodic and $h_{\mu}(T)=0$. Note that $\tilde{L}$ is an invariant set; thus $\mu(\tilde{L})=0$ or 1 for any ergodic measure. We first show that $\mu(\tilde{L})=1$ is possible.

Divide $\tilde{L}$ into $\cup_{k} \tilde{L}_{k}$ where $\tilde{L}_{k}=\{L: L$ is a line associated with $x$-coordinate $x_{1} x_{2} \ldots$ such that $k$ is the smallest integer with $x_{i} \in Q(i)$ for every $\left.i \geq k\right\}$. Then $\mu(\tilde{L})=\sum_{k} \mu\left(\tilde{L}_{k}\right)$. But $\mu\left(\tilde{L}_{k}\right)$ coincides with the measure of the projection of $\tilde{L}_{k}$ which is $\frac{|H(k-1)|}{|N(k-1)|} \prod_{i=k}^{\infty} \frac{|Q(i)|}{|N(i)|}$. Thus $\mu(\tilde{L})=\sum_{k=1}^{\infty}\left(\frac{|H(k-1)|}{|N(k-1)|} \prod_{i=k}^{\infty} \frac{|Q(i)|}{|N(i)|}\right)$ for every possible measure. Then $\mu(\tilde{L})=1$ if and only if there exists a $k$ with $\prod_{i=k}^{\infty} \frac{|Q(i)|}{|N(i)|}>0$, which is equivalent to the existence of a $k$ with $\sum_{i=k}^{\infty}\left(1-\frac{|Q(i)|}{|N(i)|}\right)<\infty$. For example, a sequence of patterns $\left\{\alpha_{i}\right\}$ with $|N(i)|=2^{i}$ and $|Q(i)|=2^{i}-1$ will yield a dynamical system having $\mu(\tilde{L})=1$.

Theorem 4.1. An Auslander system is uniquely ergodic if and only if $\mu(\tilde{L})=0$.

Proof. We need to show that $(X, T)$ is not uniquely ergodic if $\mu(\tilde{L})=1$. This can be done by defining a family of mutually singular measures on $(X, T)$. Let $0 \leq a \leq 1$ and define $f_{a}$ to be the map from the adding machine to $(X, T)$ which takes a point 
$x$ to the point $\left(x, y_{a}\right)$, where $y_{a}$ lies on the line above $x$, with proportion $a$ from the bottom of this line. If there is no line above $x$, then define $f_{a}$ in the obvious way. Then $f_{a}$ gives a measurable isomorphism between the adding machine and $\left(X, T, \mu_{a}\right)$ where the support of $\mu_{a}$ can be thought of as a cross section of $(X, T)$. Clearly $\operatorname{supp}\left(\mu_{a_{1}}\right) \neq \operatorname{supp}\left(\mu_{a_{2}}\right)$ unless $a_{1}=a_{2}$, and each $\mu_{a}$ is ergodic.

Definition 4.2. A compact dynamical system $(X, T)$ is said to be mean-L-stable if for every pair of positive numbers $\epsilon_{1}$ and $\epsilon_{2}$, there is a positive number $\delta$ such that $x, y \in X$ with $d(x, y)<\delta$ implies $d\left(T^{n} x, T^{n} y\right)<\epsilon_{1}$ for all $n$ except in a set of upper density less than $\epsilon_{2}$.

Remark. It follows from Theorem 4.1 and from results of [1] that minimal Auslander systems are uniquely ergodic if and only if they are mean-L-stable. More specifically, Theorem 8 of [1] implies that a minimal mean-L-stable system must be uniquely ergodic; and conversely, the proof given on pages 578-579 of [1] (which shows that Auslander's original example is mean-L-stable) generalizes to all Auslander systems with $\mu(\tilde{L})=0$.

We will compute the topological entropy $h(T)$ of an Auslander system $(X, T)$ as the maximum of the measure theoretic entropies $h_{\mu}(T)$.

Theorem 4.3. Let $(X, T)$ be an Auslander system. Then $h(T)=0$.

Proof. The measures $\mu_{a}$ constructed in the proof of Theorem 4.1 are the only ergodic measures on $(X, T)$. Also, $h_{\mu_{a}}(T)=0$ for all measures $\mu_{a}$. By the variational principle, $h(T)=0$.

\section{IDEMPOTENTS IN THE ENVELOPING SEMigRoup}

The enveloping semigroup of a (Z-action) dynamical system $(X, T)$ is defined as

$$
E(X, T)=E(X)=\overline{\left\{T^{n}: n \in \mathbf{Z}\right\}}
$$

where the closure is taken in the product topology. The asymptotic enveloping semigroup of $(X, T)$ is defined to be $E(X)-$ \{isolated points of $E(X)\}$ and is denoted by $H(X)$. The set of idempotents $J(X)$ in $H(X)$ is never empty (this is a consequence of what is sometimes called the Ellis theorem, see for instance [3]), and can sometimes be quite complex, as can be the enveloping semigroup itself. We can completely determine $J(X)$ for the subclass of Auslander systems having only countably many lines. The complexity of the general situation is then indicated by an example; however, the state of rigidity can be settled for all situations.

Thus we now consider minimal $(X, T)$ with the property that $|Q(k)|=1$ for every $k \geq K$, for some $K$. The analysis of $J(X)$ is simpler here because the behavior of $f \in H(X)$ on one line is mimicked by its behavior on every other line. We first define what it means for two pairs of lines to have the same relative position, then show why we can find maps $f \in H(X)$ which map all lines into certain subsets of themselves. Such maps can then be used to find the idempotents in $J(X)$.

Definition 5.1. Given four line segments $L^{1}, L^{2}, L^{3}, L^{4}$ in $X$, with lengths indicated by $l\left(L^{i}\right)$, we say that $L^{2}$ is to $L^{1}$ what $L^{4}$ is to $L^{3}$ if the following conditions hold:

a) $l\left(L^{1}\right) \cdot l\left(L^{4}\right)=l\left(L^{2}\right) \cdot l\left(L^{3}\right)$, and

b) if $b_{i}$ denotes the height of the bottom point of $L^{i}$, for $i=1, \ldots, 4$, then $\left(b_{2}-b_{1}\right) l\left(L^{3}\right)=\left(b_{4}-b_{3}\right) l\left(L^{1}\right)$. 
In the following, let $L_{1}$ denote a line of maximal length.

Lemma 5.2. Let $(X, T)$ be an Auslander system with the property $|Q(k)|=1$ for every $k$ larger than some $K$. Let $L$ be an arbitrary line in $X$. There exists a map $f \in H(X)$ such that $f$ maps $L_{1}$ linearly onto a subline of $L_{1}$ which is of the same length and at the same height as L. Furthermore, such an $f$ is unique as it must map any other line $L_{2}$ in $X$ linearly to a subline of itself satisfying $f\left(L_{2}\right)$ is to $L_{2}$ what $f\left(L_{1}\right)$ is to $L_{1}$, and take every degenerate point to itself.

Proof. Without loss of generality, we assume $l\left(L_{1}\right)=1$, so $L_{1} \sim q_{1} q_{2} \ldots$ with $q_{i} \in Q(i)$ for every $i$. Write $L \sim n_{1} n_{2} \ldots$ Let $f$ be the limit of a subsequence of $T^{m_{i}}$ where $m_{i}$ is chosen so that

$$
T^{m_{i}}\left(L_{1}\right) \sim q_{1} q_{2} \ldots q_{i} n_{1} n_{2} \ldots n_{r} q_{i+r+1} \ldots
$$

Thus

$$
m_{i}=\left(n_{1}-q_{i+1}\right) \nu_{i}+\left(n_{2}-q_{i+2}\right) \nu_{i+1}+\cdots+\left(n_{r}-q_{i+r}\right) \nu_{i+r-1} .
$$

Then $f \in H(X)$ maps $L_{1}$ as specified in the statement of the theorem. If $L_{2}$ is an arbitrary line, $L_{2} \sim s_{1} s_{2} \ldots$, then since $|Q(k)|=1$ for every $k$ larger than $K$, there exists $M$ such that $q_{m}=s_{m}$ for every $m \geq M$. So $T^{m_{i}}\left(L_{2}\right)$ eventually has the form $s_{1} s_{2} \ldots s_{i} n_{1} n_{2} \ldots n_{r} s_{r+i+1} \ldots$, and $T^{m_{i}}\left(L_{2}\right)$ is to $L_{2}$ what $L$ is to $L_{1}$. Finally, since $T^{m_{i}}$ fixes the first $i$ symbols of any expansion in $Y, f$ will map every degenerate point in $X$ to itself.

Corollary 5.3. Let $(X, T)$ be an Auslander system with the property $|Q(k)|=1$ for every $k$ larger than some $K$. If $f \in H(X)$ and $f\left(L_{1}\right)=\{(x, y)\}$ with $(x, y) \in L_{1}$, then the image under $f$ of any degenerate point in $X$ is itself and the image under $f$ of any line $L$ is the singleton $\left\{\left(x^{\prime}, y^{\prime}\right)\right\}$ where $\left(x^{\prime}, y^{\prime}\right) \in L$ and where the position of $y^{\prime}$ on $L$ is proportional to that of $y$ on $L_{1}$.

Proof. We can write $f$ as $\lim _{i \rightarrow \infty} f_{i}, f_{i} \in E(X)$, where $f_{i}\left(L_{1}\right)=L_{i} \subset L_{1}$. Then given an arbitrary line $L$ in $X$, by Lemma $5.2, f_{i}(L)$ is to $L$ what $f_{i}\left(L_{1}\right)$ is to $L_{1}$. Since $f_{i}(L) \rightarrow f(L)$, the result follows.

Now let $f_{r}$ be the element in $H(X)$ which takes $L_{1}$ to $(x, r)$, the point on $L_{1}$ of height $r$. Then Corollary 5.3 determines $f_{r}$ on the rest of $X$.

Theorem 5.4. If $\left\{\alpha_{i}\right\}$ is a sequence of subdivision patterns producing a minimal Auslander system $(X, T)$ and if there exists a $K$ such that for every $k \geq K,|Q(k)|=$ 1 , then $J(X)=\left\{f_{r}: r \in[0,1]\right\}$.

Proof. Since idempotents must project to idempotents, any idempotent in $H(X)$ must project to an idempotent on the adding machine. However, the adding machine is equicontinuous and so the only idempotent in its enveloping semigroup is the identity map. Thus if $f \in J(X)$ and $L_{1}$ is a line of maximum length, then either $f\left(L_{1}\right)=L_{1}$ or $f\left(L_{1}\right)$ is a singleton. The first case is impossible because $|Q(k)|=1$ for every $k \geq K$ implies that there are at most finitely many lines of maximum length in $X$. Thus the identity map is not in the asymptotic enveloping semigroup.

If $r$ is a dyadic rational then we can use Lemma 5.2 to find a map in $H(X)$ taking $L_{1}$ to $(x, r)$ on $L_{1}$. Since $H(X)$ is closed and since the dyadic rationals in $[0,1]$ are dense, $f_{r} \in J(X)$ for all $r \in[0,1]$. 
Note that if the system is not minimal, then one of conditions (i) or (ii) of Theorem 3.4 is violated. If there exists only finitely many $i$ with $\left|H_{U}(i)\right|>0$, then the only lines arbitrarily close to $L_{1}$ are those with bottom point having zero $y$-coordinate. This makes it impossible for any idempotent other than $f_{0}$ to be in $H(X)$. Similarly, if only finitely many $i$ have $\left|H_{L}(i)\right|>0$, then $J(X)=\left\{f_{1}\right\}$.

Next we exhibit a subdivision pattern $\left\{\alpha_{i}\right\}$ resulting in an Auslander system $(X, T)$ with the property that there exist $f \in J(X)$ and two lines $L$ and $L^{\prime}$ in $X$ both of length 1 such that $f(L)$ is a singleton on $L$ and $f\left(L^{\prime}\right)=L^{\prime}$. Thus it is possible that the set of idempotents for this system are much more varied than in the subclass discussed above. However, it is not the case that such a set is necessarily larger; in the example below it can be shown that $f_{0}$ (the map that takes every line to its bottom point) is not a member of $E(X)$. Thus not all Auslander systems satisfy $\left\{f_{r}: r \in[0,1]\right\} \subseteq J(X)$.

Let $\left\{\alpha_{i}\right\}$ be such that for every $i, N(i)=\{0,1,2,3\}, Q(i)=\{1,2\}, H_{U}(i)=\{3\}$ and $H_{L}(i)=\{0\}$. In the resulting system $(X, T)$, consider the two lines $L$ and $L^{\prime}$ given respectively by the expansions $11111 \ldots$ and $212212212 \ldots$ Let $m_{1}$ be such that $T^{m_{1}}(11111 \ldots)=11102011111 \ldots$ and inductively, let $m_{i}$ be such that $T^{m_{i}}(11111 \ldots)=n_{1} n_{2} \ldots$ where $n_{j}=1$ for the first $3 i$ symbols, then the pattern 020 is repeated for the next $3 i$ symbols and then $n_{j}=1$ for all $j$ after that. More specifically,

$$
m_{i}=\sum_{j=0}^{i-1}\left(-4^{3(i+j)+2}+4^{3(i+j)+1}-4^{3(i+j)}\right) .
$$

It is easy to check that $T^{m_{i}}(L)$ converges to the bottom point of $L$ and that $T^{m_{i}}\left(L^{\prime}\right)$ converges to $L^{\prime}$ (for instance,

$$
T^{m_{1}}\left(L^{\prime}\right)=212121212 \ldots, \quad T^{m_{2}}\left(L^{\prime}\right)=212212121121212212212 \ldots
$$

etc.). Furthermore, assuming that $T^{m_{i}}$ converges on $X$ (otherwise take a subnet), it is also easy to see that $T^{m_{i}}$ projects to the identity on the adding machine since $T^{m_{i}}$ leaves the first $3 i$ symbols fixed in the expansion of any line or point in $X$. The limit of $T^{m_{i}}$ is therefore an element of $J(X)$ taking $L$ to a singleton point on $L$ and $L^{\prime}$ to itself.

\section{Rigidity}

In [5], Glasner and Maon define three degrees of rigidity for topological systems, the two weakest being:

Definition 6.1. $(X, T)$ is weakly rigid if $\forall \epsilon>0$ and points $z_{1}, z_{2}, \ldots z_{n} \in X$, $\exists k \in \mathbf{Z}-\{0\}$ such that $d\left(T^{k} z_{i}, z_{i}\right)<\epsilon(i=1, \ldots, n)$.

Definition 6.2. $(X, T)$ is rigid (w.r.t. some sequence $\left.n_{k} \nearrow \infty\right)$ if $T^{n_{k}} z \rightarrow z$ $\forall z \in X$.

Clearly Def. 6.1 is equivalent to

Definition 6.3. $(X, T)$ is weakly rigid if $i d_{X} \in H(X)$.

Glasner and Maon show that the topological entropy of a rigid flow is zero. Note that we have shown in section 4 that the topological entropy of any Auslander system is zero. We show in this section that Auslander systems fail to even be weakly rigid. Indeed, despite the complexity of $J(X)$ for a general Auslander system, we can still prove that $i d_{X}$ is never in $H(X)$.

Recall that $T$ acts on the first coordinate as the adding machine. For $\bar{x}=$ $x_{1} x_{2} \ldots$, let us write $\overline{T x}=\left(x_{1}+1\right) x_{2} \ldots$ Then $\overline{T^{\nu_{1}} x}=x_{1}\left(x_{2}+1\right) x_{3} \ldots, \overline{T^{\nu_{j}} x}=$ 
$x_{1} \ldots x_{j}\left(x_{j+1}+1\right) x_{j+2} \ldots$, etc. Given a positive integer $n$, we can write $n$ uniquely as $c_{0}+c_{1} \nu_{1}+\cdots+c_{j} \nu_{j}$, and $\overline{T^{n} x}=\left(x_{1}+c_{o}\right)\left(x_{2}+c_{1}\right) \ldots\left(x_{j+1}+c_{j}\right) x_{j+2} \ldots$ All sums must be taken mod $n_{j}$ and there might be 'carrying over' from one index to the next.

Theorem 6.4. Auslander systems are not weakly rigid.

Proof. Assume there are infinitely many $k$ with $|Q(k)|>1$ (otherwise section 5 gives the result). We will assume $i d_{X} \in H(X)$ and reach a contradiction.

If $i d_{X} \in H(X)$ then given two lines $L_{1}, L_{2}$ and arbitrary $\epsilon$, there must be $m$ such that $T^{m}\left(L_{i}\right)$ is within $\epsilon$ of $L_{i}, i=1,2$. Assume $m>0$ (else switch the roles of $L_{1}$ and $L_{2}$ in the following argument). Pick the two lines to be

$$
L_{1} \sim l_{11} l_{12} \ldots, \quad L_{2} \sim l_{21} l_{22} \ldots
$$

such that $l_{1 i}$ is the smallest element in $Q(i)$ and $l_{2 i}$ the largest. (It is enough to take this for large enough $i$.) There could be $i$ such that $l_{1 i}=l_{2 i}$, but there are infinitely many $i$ where they are not equal, by assumption.

Write $m$ as $c_{j} \nu_{j}+c_{j+1} \nu_{j+1}+\cdots+c_{p} \nu_{p}$ where $c_{j} \neq 0$. We know

$$
T^{c_{j} \nu_{j}}\left(L_{1}\right) \sim l_{11} \ldots l_{1 j}\left(l_{1(j+1)}+c_{j}\right) \ldots
$$

and

$$
T^{c_{j} \nu_{j}}\left(L_{2}\right) \sim l_{21} \ldots l_{2 j}\left(l_{2(j+1)}+c_{j}\right) \ldots
$$

For $m$ sufficiently large, $l_{1(j+1)}+c_{j}$ and $l_{2(j+1)}+c_{j}$ must be elements in $Q(j+1)$. Since $c_{j}>0$, there must be carrying over in $T^{c_{j} \nu_{j}}\left(L_{2}\right)$, i.e.,

$$
T^{c_{j} \nu_{j}}\left(L_{2}\right) \sim l_{21} \ldots l_{2 j}\left(l_{2(j+1)}+c_{j}\right)\left(l_{2(j+2)}+1\right) \ldots
$$

Now there are two possibilities:

a) If $B_{j+2}^{\nu_{j+2}-1}$ is of type $\mathrm{H}$, then $l_{2(j+2)}+1 \notin Q(j+2)$ and $c_{j+1}$ must be nonzero. Thus $m$ includes the summands $c_{j} \nu_{j}$ and $c_{j+1} \nu_{j+1}$. Note

$$
T^{c_{j} \nu_{j}+c_{j+1} \nu_{j+1}}\left(L_{2}\right) \sim l_{21} \ldots l_{2 j}\left(l_{2(j+1)}+c_{j}\right)\left(l_{2(j+2)}+1+c_{j+1}\right) \ldots
$$

and we again have carrying over, now in the $j+2$ digit. Thus

$$
T^{c_{j} \nu_{j}+c_{j+1} \nu_{j+1}}\left(L_{2}\right) \sim l_{21} \ldots l_{2 j}\left(l_{2(j+1)}+c_{j}\right)\left(l_{2(j+2)}+1+c_{j+1}\right)\left(l_{2(j+3)}+1\right) \ldots
$$

b) If $B_{j+2}^{\nu_{j+2}-1}$ is of type $\mathrm{F}$, then $l_{2(j+2)}=n_{j+2}-1$ and whether $c_{j+1}$ is zero or not, we have carrying over in the $j+2$ term.

Since there is carrying over in the next digit, we can repeat the above: either $c_{j+2}$ is zero or not, but either way, there is carrying over to the next term. This process never stops and we know a) occurs infinitely many times. Thus $m$ contains infinitely many $c_{i} \neq 0$, which contradicts it being finite.

\section{REFERENCES}

[1] J. Auslander, Mean-L-stable systems, Illinois Journal of Math., 1959, pp. 566-579. MR 26: 6950

[2] J. Auslander and K. Berg, A condition for zero entropy, Israel J. of Math.,vol. 69, 1990, pp. 59-64. MR 91d:54045

[3] R. Ellis, Lectures on Topological Dynamics, W. A. Benjamin, 1969. MR 42:2463 
[4] E.E. Floyd, A non-homogeneous minimal set, Bull. Amer. Math. Soc.,vol. 55, 1949, pp. 957960 MR 11:453g

[5] S.Glasner and D.Maon, Rigidity in topological dynamics, Ergodic Theory and Dynamical Systems,vol. 9, 1989 MR 90h:54050

Department of Mathematics, California State University at Bakersfield, BakersFIELD, CALIFornia 93311

E-mail address: khaddad@ultrix6.cs.csubak.edu

Department of Mathematics \& Statistics, Swarthmore College, Swarthmore, PennSYLVANIA 19081

E-mail address: aimee@swarthmore.edu 